\title{
A High-Performance Infrared Imaging System with Adaptive Contrast Enhancement
}

\author{
Feng DENG ${ }^{\text {a }}$, Zhong SU ${ }^{\text {a }}$, Rui WANG ${ }^{\mathrm{b}}$, Jun $\mathrm{LIU}^{\mathrm{c}, \mathrm{d}, 1}$ and Yanzhi WANG ${ }^{\mathrm{c}}$ \\ ${ }^{a}$ Beijing Key Laboratory of High Dynamic Navigation Technology, Beijing Information \\ Science \& Technology University, Beijing, China \\ ${ }^{b}$ State Key Laboratory of Nickel and Cobalt Resources Comprehensive Utilization, \\ Jinchang, China \\ ${ }^{c}$ Robotics Institute, School of Computer Science, Carnegie Mellon University, \\ Pittsburgh, PA, USA \\ ${ }^{d}$ Department of Electrical \& Computer Engineering, College of Engineering, \\ Northeastern University, Boston, MA, USA
}

\begin{abstract}
Most of the existing infrared imaging systems employ the scheme of FPGA/FPGA+DSP with numerous peripheral circuits, which leads to complex hardware architecture, limited system versatility, and low computing performance. It has become an intriguing technical problem worldwide to simplify the system structure while improving the imaging performance. In this paper, we present a novel real-time infrared imaging system based on the Rockchip's RV1108 visual processing $\mathrm{SoC}$ (system on chip). Moreover, to address the problem of low contrast and dim details in infrared images with a high dynamic range, an adaptive contrast enhancement method based on bilateral filter is proposed and implemented on the system. First, the infrared image is divided into a base layer and a detail layer through bilateral filter, then the base layer is compressed by an adaptive bi-plateau histogram equalization algorithm, and finally a linear-weighted method is used to integrate the detail layer to obtain the image with enhanced details. The experimental results indicate that compared with traditional algorithms, our method can effectively improve the overall contrast of the image, while effectively retaining the image details without noise magnification. For an image of $320 * 240$ pixels, the real-time processing rate of the system is 68 frames/s. The system has the characteristics of simplified structure, perceptive image details, and high computing performance.
\end{abstract}

Keywords. Infrared imaging system, RV1108, real-time, contrast enhancement, bilateral filter, bi-plateau histogram equalization, high dynamic range

\section{Introduction}

The infrared (IR) imaging system converts the temperature distribution of the target object into a human visual distinguishable image by non-invasive detection of the object infrared radiation using infrared thermography technology. With innovative

\footnotetext{
${ }^{1}$ Corresponding Author, Jun Liu, Robotics Institute, School of Computer Science, Carnegie Mellon University, 4227 Newell-Simon Hall, 5000 Forbes Avenue Pittsburgh, PA 15213, USA; E-mail: junliu2@andrew.cmu.edu.
} 
breakthroughs in infrared technology, the application of IR imaging systems has expanded from the military to various civilian industries, which is playing an increasingly important role nowadays. Research on high-performance IR imaging systems is particularly urgent.

Since the first forward-looking infrared imaging system was invented in the United States in the 1960s [1], the infrared thermal imaging system has evolved from the first generation to the third generation $[2,3]$. However, the FPGA/FPGA+DSP system structure with plenty of peripheral circuits is widely used for the image processing in most of the existing IR systems, which leads to complex hardware architecture, limited system versatility, and low computing performance. On the other hand, IR images are prone to loss of local detail information when adapt data from the detector to the display. The two main reasons for this problem are: (1) Limited by the constraints of the IR detector manufacturing process, the original IR image has the characteristics of low contrast, blurred details and poor signal-to-noise ratio; (2) The IR detector's response output is 14bits or 16bits for high-performance IR imaging system, which cannot be managed by the general 8 bits display devices. Therefore, in the process of the high dynamic range compression, detailed textures with small background temperature differences in the image are vulnerable to lost. It has become an intriguing technical problem worldwide to optimize the system structure while enhancing computation performance and improving details of potential or weak targets in high dynamic range IR images in new generation IR imaging system.

Traditional infrared image enhancement algorithms usually use methods such as automatic gain control (AGC) [4] and histogram equalization (HE) [5, 6], which can better adjust the contrast information of the image, but it is prone to "over bright" or "over dark" in local areas, and lose small details in raw images. The image layer based and transform domain based approaches have better contrast enhancement compared to AGC and HE. F. Branchitta [7] presented a detail enhancement algorithm BF\&DRP for high dynamic range compression based on bilateral filtering. C. Zuo et al. [8] proposed an improved method BF\&DDE, which using adaptive Gaussian filtering to avoid gradient reversal artifacts caused by the over enhancement of the detailed layer. N. Liu et al. [9] presented a method based on guided filtering to get better contrast enhancement. However, there are many parameters needed to be carefully tuned in the image layer based algorithms for the specific scenario so as to take on the best visualization effect. Transform-domain based approach [10,11] and AI (artificial intelligence) $[12,13]$ based method can effectively enhance the small details in the target scene of the IR image, but the algorithms have high time consuming and space complexity, which is not conducive to implementation on embedded systems with limited system resources and high realtime requirements.

In this paper, we present a novel real-time infrared imaging system based on the Rockchip's RV1108 visual processing SoC (system on chip). And moreover, to address the problem of low contrast and dim details in infrared images with a high dynamic range, an adaptive contrast enhancement method based on bilateral filter is proposed and implemented on the system. Our system surpasses traditional IR imaging systems in terms of system structure, imaging quality and real-time performance.

The rest of this paper is organized as follows. Section 2 describes the system architecture in brief. Section 3 elaborates on adaptive contrast enhancement method. Section 4 presents our experimental results and evaluation. Section 5 concludes the paper with remarks on our future work. 


\section{System architecture design}

Infrared imaging system is generally composed of optical system, infrared detector, AD conversion circuit and signal processor. In our proposed system, a continuous zoom infrared germanium lens is used as optical lens, an uncooled focal plane array with far infrared band of 8-14 microns is employed as infrared detector, and Rockchip's RV1108 is adopted as main image processor of the system.

RV1108 is a professional visual processing SoC, which embeds a new-generation DSP for digital processing and an ARM Cortex-A7 single-core processor for system scheduling and application. The ARM microprocessor is designed for system configuration, framework management, DSP driver, and I/O communication interaction. DSP in RV1108 is in charge of executing various image processing algorithms with high efficiency by utilizing specific DSP optimization techniques. Critical system and algorithm codes are stored in DSP internal memories to speed up the program execution with low latency. In addition, RV1108 is capable of supporting H.264 video encoder/decoder up to $1440 \mathrm{p} @ 30 \mathrm{fps}$, simultaneously inputting up to 4 cameras, and displaying on one screen [14]. It also supports various camera interfaces such as MIPI (mobile industry processor interface)-CSI (camera serial interface), CVBS (composite video broadcast signal) in, and lots of display interfaces such as MIPI-DSI (display serial interface), HDMI (high definition multimedia interface) 1.4, CVBS out, and serial/parallel RGB. Since RV1108 is a highly integrated and cost-effective SoC, it can fully meet the requirements of a high-performance IR imaging system in terms of efficiency, power consumption, and cost-effectiveness. The block diagram of the system hardware architecture is shown in Fig.1.

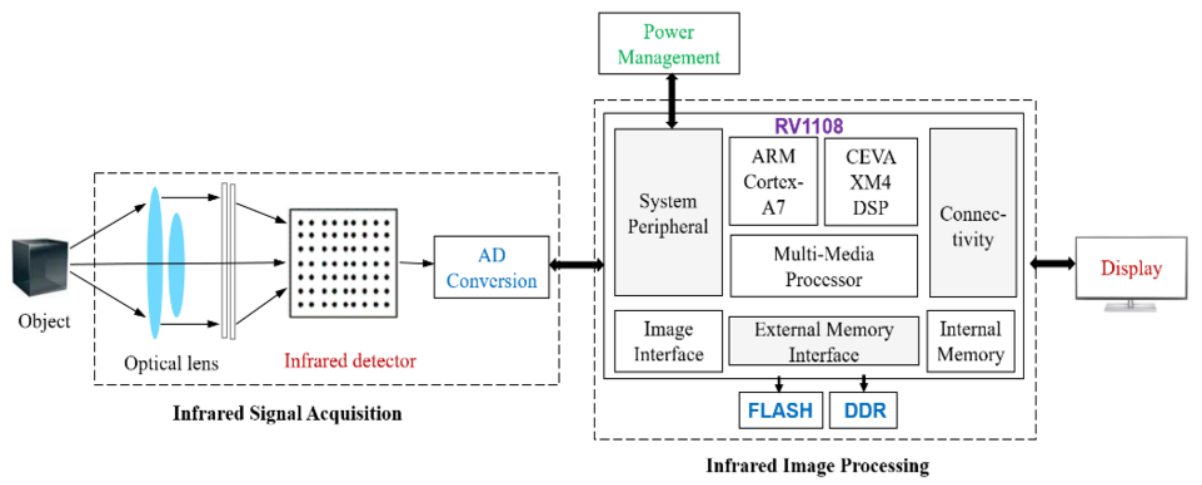

Fig. 1 Block diagram of the system architecture

\section{Proposed method}

The original input IR images with a wide dynamic range of 14-bit (or more) results in the invisible or faint details visualization during the high dynamic compressing process. Inspired by the visualized effect of BF\&DRP method, we propose an adaptive contrast enhancement method, which can adaptively adjust the algorithm parameters so as to achieve the best visualization performance in different scenarios. Fig.2 shows the flowchart of our algorithm. First, bilateral filter is adopted to decompose the image into 
the base component and the detail component, which corresponds to the base layer and the detail layer, respectively. Then, an adaptive bi-plateau histogram equalization method is performed on the base layer to dynamically enhance the image contrast according to the image grayscale in different scenarios. At last, we integrate the two parts by linear-weighted method and quantize the result to 8-bit display range.

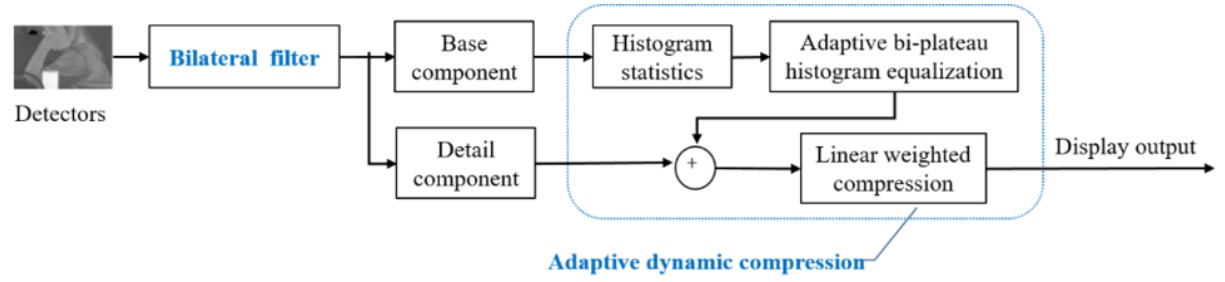

Fig. 2 Flowchart of adaptive contrast enhancement algorithm

\subsection{Bilateral filter}

Bilateral filter can be acknowledged as a successful filter unlike traditional filters for edge preservation and noise elimination. Using a Gaussian $G_{\sigma}$ as a decreasing function, and considering a gray-level image $I$, the result $I^{b}$ of the bilateral filter is defined by [15]:

$$
\begin{gathered}
I_{p}^{b}=\frac{1}{W_{p}^{b}} \sum_{q \in S} G_{\sigma_{S}}(\|p-q\|) G_{\sigma_{r}}\left(I_{p}-I_{q}\right) I_{q} \\
\text { with } W_{p}^{b}=\sum_{q \in S} G_{\sigma_{S}}(\|p-q\|) G_{\sigma_{r}}\left(I_{p}-I_{q}\right)
\end{gathered}
$$

where $S$ denotes the spatial domain, $I_{p}$ is the gray value of image $I$ at position $p$, the parameter $\sigma_{s}$ defines the size of the spatial neighborhood used to filter a pixel, and $\sigma_{r}$ controls how much an adjacent pixel is down weighted because of the intensity difference. $W^{b}$ normalizes the sum of the weights.

Fig. 3 shows the detail layer images obtained using bilateral filter with different filtering windows $r$. It can be seen, as the filtering window decreases, the detail edges after bilateral filtering become clearer under a certain $\sigma_{s}$ and $\sigma_{r}$.

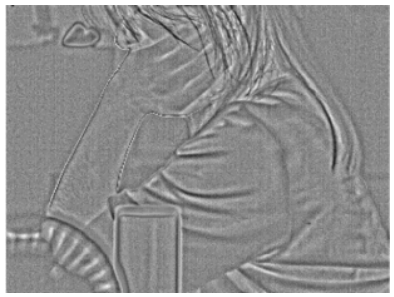

(a)

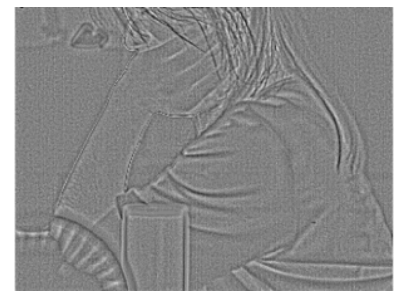

(b)

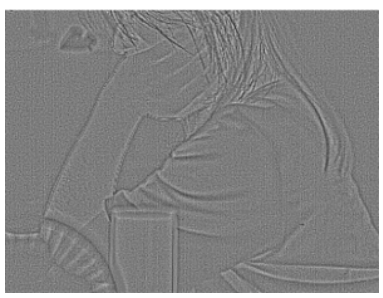

(c)

Fig. 3 Detail layer image using bilateral filter with different filtering window. (a) $\mathrm{r}=8$, $\sigma_{s}=4, \sigma_{r}=0.01$. (b) $\mathrm{r}=4, \sigma_{s}=4, \sigma_{r}=0.01$. (c) $\mathrm{r}=2, \sigma_{s}=4, \sigma_{r}=0.01$. 


\subsection{Adaptive dynamic compression}

After bilateral filtering, we get base component of image as base layer. Then, the subtraction of the input image and the base component is then determined as the detail layer. Since the detail component of the image occupies a smaller dynamic range than the base component, if the dynamic range compression is performed on a uniform scale, the great effect of detail enhancement cannot be achieved. Therefore, we process each component separately. For base layer, the contrast is relatively low, and the image is dark with less details, we propose an adaptive bi-plateau histogram equalization (ABPHE) to enhance the contrast of background. Then integrate the base layer and detail layer by the linear-weighted method to display range. The proposed algorithm can constrain the overbright background and noises by setting an upper plateau threshold, and enhance the image details and magnify the dim targets by setting a lower threshold value. The upper and lower threshold value can be adjusted adaptively according to different scenarios.

The method of calculating the upper plateau threshold $T_{u p}$ is as follows:

$$
T_{u p}=\operatorname{mean}\{\operatorname{POLAR}(1), \operatorname{POLAR}(k), \ldots, \operatorname{POLAR}(P)\}
$$

where POLAR is the set of local maximums of the histogram with zero statistics removed, and $\mathrm{P}$ is its size. POLAR(k) is the kth element in POLAR.

The calculation of local maximums depends on the value of the window length of the histogram, and enhancement performance will be affected by the calculation result of the upper plateau threshold. According to the experiment, the value of window length 20 is recommended.

The method of calculating the lower plateau threshold is describe as pseudocode shown in Algorithm 1.

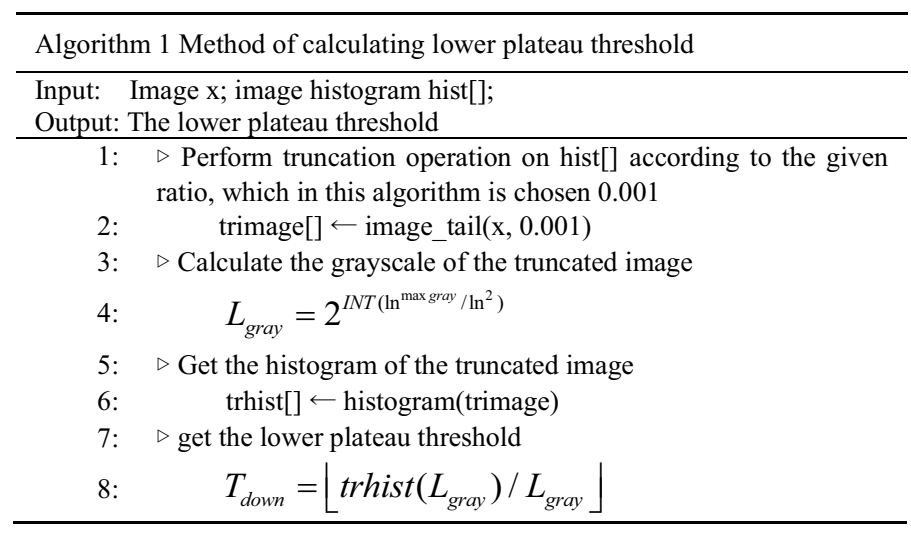

Fig. 4 shows the result comparison of base layer by HE and ABPHE. It is shown that some local areas turn out to be "over bright" in the base layer after processed by HE, such as building outline, pedestrian profile, and tree leaves. While the base layer has uniform brightness and good overall contrast by ABPHE. 


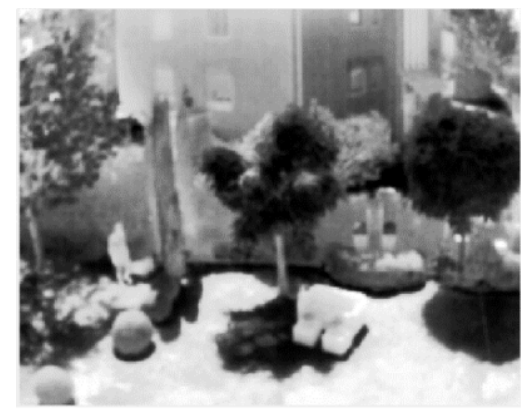

(a) The base layer by HE

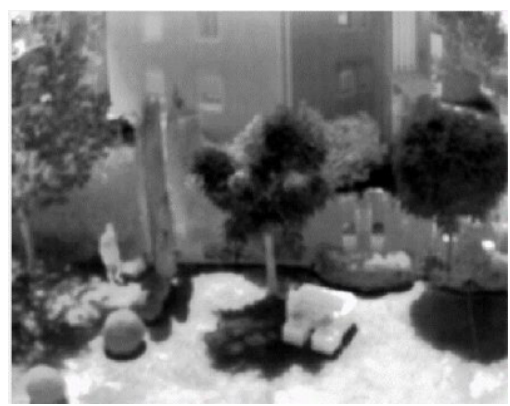

(b) The base layer by ABPHE

Fig.4 The result comparison of base layer by HE and ABPHE

\subsection{Recombine of the base and detail layer}

The base layer is projected into the proper range using ABPHE method. In order to display the enhanced image onto the general monitor devices with 8 bits, we first map the processed base layer and the detail layer into the gray scale (typically 0 to 255) by linear strength method. The processed base and detail components are represented by $I_{b a}$ and $I_{d e}$. Finally, the two components are recombined by linear-weighted algorithm to get the final output image $I_{\text {out }}$.

$$
I_{\text {out }}=\alpha * I_{b a}+(1-\alpha) * I_{d e}
$$

where $\alpha(0<\alpha<1)$ is weighted coefficient. The large the $\alpha$, the less the detail information in the image. We empirically set $\{\alpha \mid 0.3 \leq \alpha \leq 0.5\}$.

\section{Experimental results and performance evaluation}

\subsection{System prototype based on RV1108}

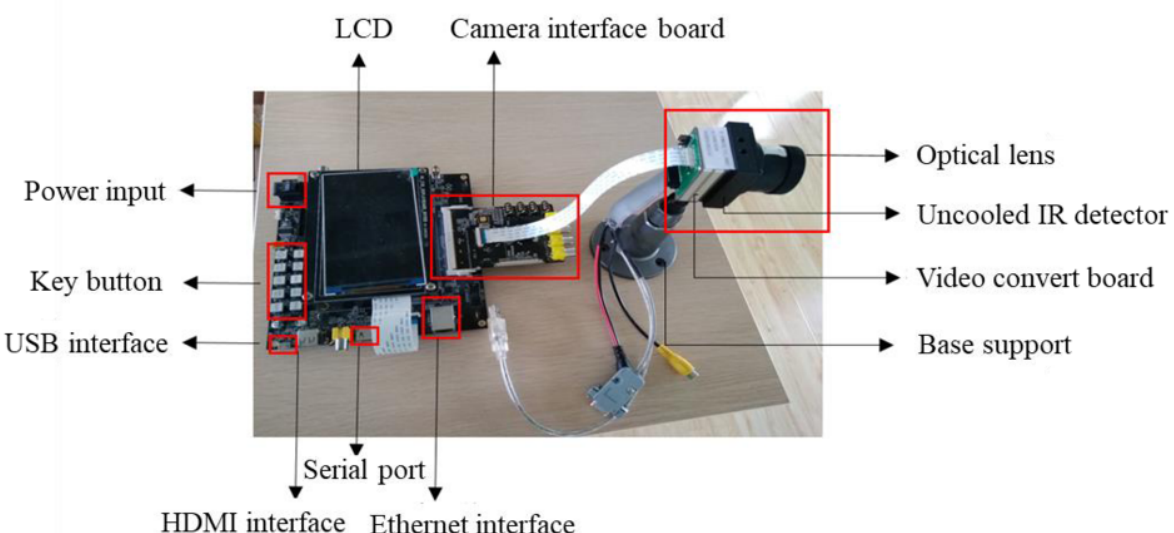

Fig.5 Infrared imaging system prototype based on RV1108 
The IR imaging system prototype based on RV1108 developed in this paper is shown in Fig.5. The system uses $8-14 \mu \mathrm{m}$ uncooled IR focal plane detector, the resolution of the video image collected by the system is $320 \times 240$ pixels, and the accuracy of the raw image is 14-bit.

We ported and implemented the adaptive contrast enhancement method onto the prototype system. To evaluate the system performance, we choose two sets of image data in different scenarios, as shown in Fig.6 (a) and Fig.7 (a). Fig.6 (a) was collected by the proposed IR system. Fig.7 (a) was provided by FIIR with 16-bit data width and resolution $324 * 256$ pixels. The proposed method was compared with the popular image enhancement algorithms, i.e., AGC, HE and BF\&DRP.

\subsection{Subjective tests}

The comparison results of different algorithms and our algorithm is shown in Fig.6 and Fig.7. The experimental results illustrate that the proposed method provides the best visual effect and contrast enhancement over all two sets of images. Besides, it effectively retains the detail information and avoids amplification of the noise.

Fig. 6 and Fig.7 provide a high dynamic range scenario with abundant details. It is noticeable that image by AGC has low contrast and less details, image by HE can map the contrast to a certain range, but has the "over bright" phenomenon. For example, the hot cup and girl fingers in Fig.6 (b)), the building in Fig.7 (b) are over-enhanced and halos are produced around the cup, building outline and tree leaves. The method of BF\&DRP can improve the overall contrast while maintaining the details of the image, but it tend to magnify the noise in flat regions, for example, the noise in the background are highlighted shown in the Fig.6 (c) and Fig.7 (c). Our proposed method achieves the best performance in terms of contrast enhancement, details perception, and noise suppression. It can be seen evidently from Fig. 6 (d) and Fig.7 (d) that the overall contrast has enhanced, and some details obscure in the original images have visualized, such as the girl's hair, fingers, clothes folds, edge of hot cup, fan wheel, pedestrian and tree shadow etc. Besides, our method is free of artifacts, producing a natural and homogeneous image without tuning parameters among different scenarios.

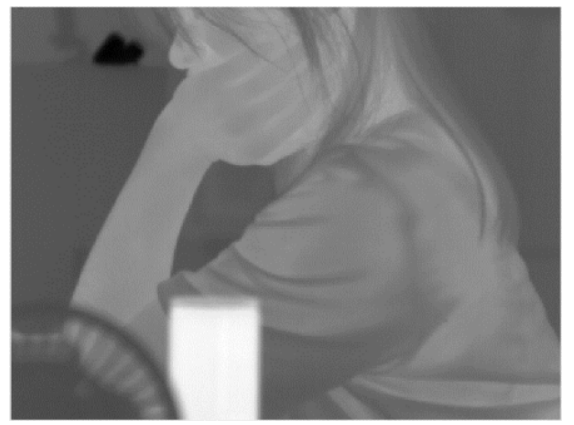

(a) AGC

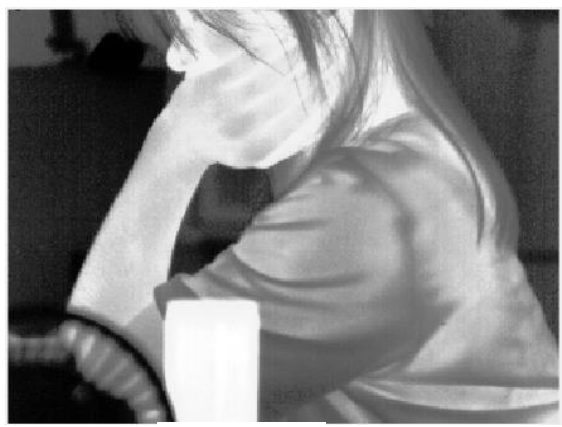

(b) $\mathrm{HE}$ 


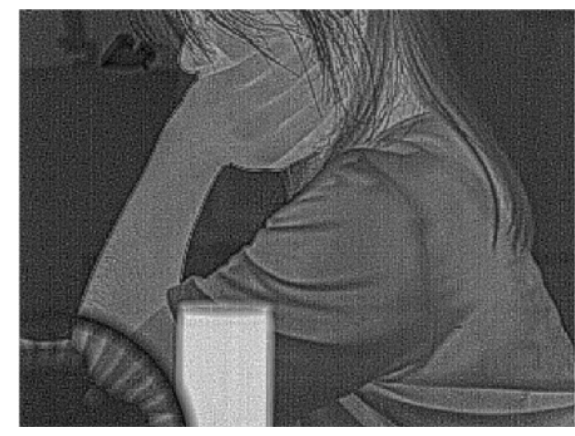

(c) BF\&DRP

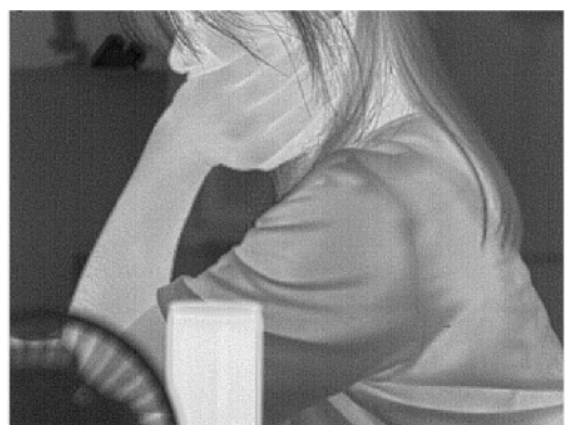

(d) Proposed method

Fig.6 Comparison results of scene 1

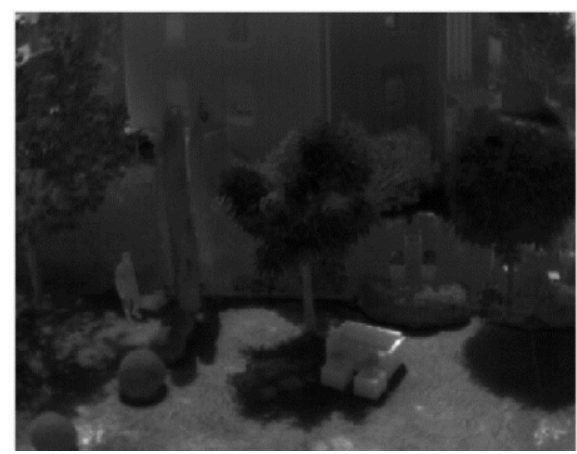

(a) AGC

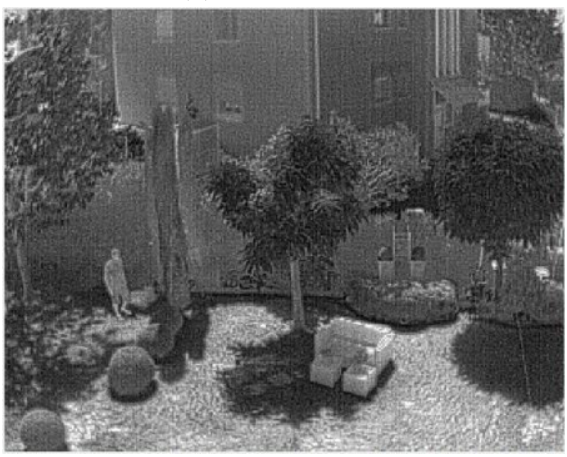

(c) BF\&DRP

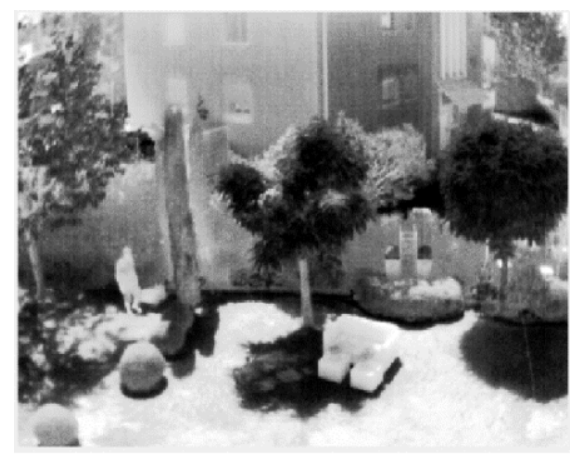

(b) HE

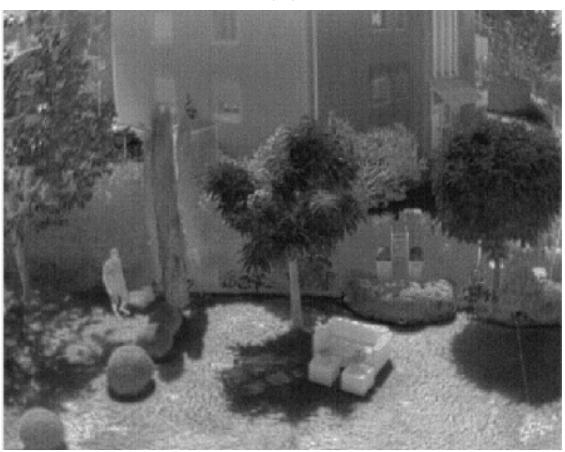

(d) Proposed method

Fig.7 Comparison results of scene 2

\subsection{Quantitative tests}

In the quantitative tests, standard deviation (STD) and peak signal to noise ratio (PSNR) are used to objectively evaluate the image enhancement effect of different methods. The 
test results are given in Table 1. It should point out that HE has higher STD value than BF\&DRP and the proposed method, but its PSNR is too low to achieve satisfied visual effects. Compare with BF\&DRP, the proposed method has higher STD and PSNR, which is proven to be more effective on image contrast enhancement.

Table 1 The objective evaluation indicators of different algorithms

\begin{tabular}{ccccc}
\hline & Scenario & HE & BF\&DRP & Proposed \\
\hline \multirow{2}{*}{ STD } & Fig.6 & 73.6742 & 38.0261 & 51.9192 \\
& Fig.7 & 73.7171 & 41.1081 & 43.3585 \\
PSNR & Fig.6 & 14.8819 & 16.6070 & 20.0997 \\
& Fig.7 & 8.1422 & 13.3014 & 15.0134 \\
\hline
\end{tabular}

\subsection{Computation performance}

To further demonstrate the system computation performance, we experiment the running time of our methods on the system prototype platform. Table 2 presents the performance evaluation of the core algorithms in terms of RV1108 cycles occupation, average execution time and quantity of code. For the image with size of $320 * 240$ pixels, the average execution time of imaging enhancement algorithm is $14.50 \mathrm{~ms} /$ frame under the RV1108 DSP frequency of $600 \mathrm{MHz}$, namely the image processing speed of the system is 68 frames/s. The evaluation results illustrate that our algorithms have very low time/space complexity, and the real-time performance of the system is excellent and competitive.

Table 2 Computation performance evaluation

\begin{tabular}{cccc}
\hline Core algorithms & $\begin{array}{c}\text { RV1108 cycles } \\
(\mathrm{M} / \text { frame })\end{array}$ & $\begin{array}{c}\text { Average } \\
\text { execution time } \\
(\mathrm{ms} / \text { frame })\end{array}$ & $\begin{array}{c}\text { Code quantity } \\
(\mathrm{KB})\end{array}$ \\
\hline Normalization & 0.046 & 0.076 & 0.542 \\
Bilateral filter & 3.266 & 5.444 & 4.654 \\
Histogram statistics & 0.547 & 0.912 & 0.384 \\
Bi-plateau histogram & 1.789 & 2.997 & 3.202 \\
equalization & 7.668 & 12.779 & 1.952 \\
\hline
\end{tabular}

\section{Conclusion}

In this paper, we focused on designing a high-performance IR imaging system with stateof-art contrast enhancement method. Most of the previous works on IR imaging system are based on FPGA/FPGA+DSP architecture, which leads to complex system architecture and limited computing performance. Aiming at these issues, we designed a high-performance uncooled IR imaging system based on the Rockchip RV1108 visual processing $\mathrm{SoC}$ to increase the system integration and reliability. Moreover, to address 
the problem of low contrast and dim details in infrared images with a high dynamic range, an adaptive contrast enhancement method based on bilateral filter is proposed and implemented on the system. The experimental results indicate that compared with traditional algorithms, our method can effectively improve the overall contrast of the image, while effectively retaining the image details without noise magnification. The system has the characteristics of simplified structure, perceptive image details, and high computing performance. In the future, we will further verify the adaptability of our algorithm in various scenarios, so as to enhance the robustness of the presented IR imaging system.

\section{ACKNOWLEDGMENT}

This work was supported in part by National Natural Science Foundation of China (41871348); Beijing Information Science \& Technology University (2020KYNH224); and Beijing Key Laboratory of High Dynamic Navigation Technology (HDN2019002).

\section{REFERENCES}

[1] A. Rogalski. Infrared detectors: an overview [J]. Infrared Physics \& Technology, 2002, 43 (3-5): 187210. http://dx.doi.org/10.1016/s1350-4495(02)00140-8.

[2] A. Rogalski. Progress in focal plane array technologies [J]. Prog. Quantum Electron, 2012, 36 (2-3): 342-473. http://dx.doi.org/10.1016/j.pquantelec.2012.07.001.

[3] P. Martyniuk, J. Antoszewski, M. Martyniuk, L. Faraone, A. Rogalski. New concepts in infrared photodetector designs [J]. Appl. Phys. Rev. 1 (4) (2014) 041102. http://dx.doi.org/10.1063/1.4896193.

[4] R. C. Gonzalez, R. E. Wood. Digital Image Processing [M]. $2^{\text {nd }}$ ed. Upper Saddle River: Prentice-Hall, 2002.

[5] H.O. Chen, N.S.P. Kong, H. Ibrahim. Bi-histogram equalization with a plateau limit for digital image enhancement [J]. IEEE Transactions on Consumer Electronics, 2010, 55(4): 2072-2080.

[6] S. Agrawal, R. Panda, P. K. Mishro. A novel joint histogram equalization based image contrast enhancement [J]. Journal of King Saud University- Computer and Information Sciences, 2019: 1-11.

[7] F. Branchitta, M. Diani, G. Corsini. New technique for the visualization of high dynamic range infrared images source [J]. Optical Engineering, 2009, 48(9): 1-9.

[8] C. Zuo, Q. Chen, J. Ren. Display and detail enhancement for high-dynamic-range infrared images [J]. Optical Engineering, 2011, 50(12): 895-900.

[9] N. Liu, D. Zhao. Detail enhancement for high-dynamic-range infrared images based on guided image filter [J]. Infrared Physics \& Technology, 2014, 67: 138-147.

[10] H. I. Ashiba, H. M. Mansour, et al. Enhancement of IR images using histogram processing and the undecimated additive wavelet transform [J]. Multimedia Tools and Applications, 2019, 78: 11277-11290.

[11] Y. Chang, M. Chen, et al. Toward universal stripe removal via wavelet-based deep convolutional neural network. IEEE Transactions on Geoscience and Remote Sensing, 2019, 58(4): 2880-2897

[12] Z. He, Y. Cao, Y. Dong, et al. Single-image-based nonuniformity correction of uncooled long-wave infrared detectors: a deep-learning approach [J]. Applied Optics, 2018, 57(18): 155-164.

[13] F. Lv, B. Liu, F. Lu. Fast Enhancement for Non-Uniform Illumination Images using Light-weight CNNs. ACM Multimedia 2020: 1450-1458

[14] Fuzhou Rockchip Electronics Co., Ltd. Rockchip RV1108 Datasheet (Revision 1.4). June 2018.

[15] Tseng Y C, Hsu P H, Chang T S. A 124 Mpixels/s VLSI Design for Histogram-Based Joint Bilateral Filtering [J]. IEEE Transactions on Image Processing, 2011, 20(11): 3231-3241. 